\title{
Video absorbtiometry in diagnostic cytology: Description of a new technique and a preliminary statistical evaluation
}

\author{
J A Lee, D J Spargo, P A Riley
}

\begin{abstract}
Cytological specimens from 21 patients were examined using digital video microscopy. The staining characteristics of cells incubated with hydroquinone and the tetrazolium stain MTT were analysed using a novel procedure that is independent of morphological features and relies on characteristics of the frequency distribution of the localised (pixel) absorbances of the cellular images. The properties of the distribution of absorbances were assessed by a number of procedures. Comparisons were made of nine malignant and 12 non-malignant cytological samples, obtained mainly from pleural and peritoneal fluid. The data showed significant differences between the sets of samples which may be of value in cytological diagnosis. In the material examined all the malignant samples exhibited a positive skewness with the $\alpha-3$ test (the mean $\alpha-3$ value being greater than 0.5); this was true in only two of 12 non-malignant samples.

This analytical technique, which is largely independent of morphology, magnification, and absolute absorbance values, seems to hold promise for the simplification and automation of cytological screening procedures.
\end{abstract}

The importance of cytological screening in the early detection of certain cancers has stimulated interest in the possibility of using automatic screening procedures to exclude the large numbers of cytological specimens that are normal. A great deal of effort has therefore gone into devising discriminatory diagnostic tests for malignant or pre-malignant changes, and recently there have been important developments in the use of multivariate analysis and expert systems for this purpose. ${ }^{12}$ One problem of this approach is that it demands considerable computing time and requires technically advanced and expensive apparatus. This is because many of the diagnostic features that are recognisable by experienced cytologists are spatial and textural in nature, and these are difficult to quantify simply. In this study we examined the possibility of using featureless cytological criteria, which are independent of morphology, and hence easier to define and manipulate. Point absorbance measurements of cells were used to construct frequency distributions, the characteristics of which can be readily quantified by calculated parameters.

\section{Methods}

The specimens examined were obtained from 21 patients. Slides were prepared in parallel with the routine cytological material. Air dried samples were fixed with formaldehyde $(10 \%)$ and stained in batches by incubation for 30 minutes at $37^{\circ} \mathrm{C}$ in a solution containing per $100 \mathrm{ml}: 25 \mathrm{ml}$ 3-(4,5 dimethylthiazol-2-yl)2,5-diphenyl-tetrazolium bromide (MTT) (Sigma, Poole, Dorset), $1 \mathrm{mg} / \mathrm{ml}$ in distilled water; $5 \mathrm{ml}$ cobalt chloride $0.5 \mathrm{~mol} / 1 ; 40 \mathrm{ml}$ TRIS buffer $0.2 \mathrm{~mol} / 1, \mathrm{pH} 7 \cdot 4 ; 10 \mathrm{ml}$ hydroquinone (Sigma) $40 \mathrm{mg} / \mathrm{ml}$ in TRIS; $20 \mathrm{ml}$ distilled water and $200 \mathrm{mg}$ catalase (Sigma). ${ }^{3}$ This staining procedure is believed to detect sites of redox activity due to ubiquinones, naphthoquinones, and related compounds. ${ }^{3-5}$ Ubiquinones are an important constituent of mitochondria and abnormalities in mitochondrial biogenesis are an early feature of malignant change. ${ }^{67}$

After staining, the material was washed and mounted in neutral glycerin jelly for inspection. Measurements of at least 60 cells per sample were made, using a Zeiss photomicroscope fitted with an Hitachi KP4 video camera connected to an Intellect 200 Image Analysing system interfaced to a PDP $11 / 23+$ host computer. Luminosity data were collected at $540 \mathrm{~nm}$ using a narrow band interference filter

Table 1 Diagnosis of each specimen using standard cytological techniques

\begin{tabular}{lll}
\hline Sample & & \\
No & Source & Cytological diagnosis \\
\hline 1 & Pleural fluid & Adenocarcinoma \\
2 & Laparotomy wash & Negative \\
3 & Pleural fluid & Adenocarcinoma \\
4 & Ascitic fluid & Adenocarcinoma \\
5 & Pleural fluid & Negative \\
6 & Pleural fluid & Adenocarcinoma \\
7 & Pleural fluid & Adenocarcinoma \\
8 & Pleural fluid & Negative \\
9 & Pleural fluid & Negative (lymphocytic) \\
10 & Pleural fluid & Negative (lymphocytic) \\
11 & Pleural fluid & Negative (lymphocytic) \\
12 & Ascitic fluid & Negative \\
13 & Ascitic fluid & Negative \\
14 & Pleural fluid & Mesothelioma \\
15 & Pleural fluid & Negative \\
16 & Pleural fluid & Negative \\
17 & Pleural fluid & Negative \\
18 & Ascitic fluid & Adenocarcinoma \\
19 & Pleural fluid & Negative \\
20 & Pleural fluid & Malignant \\
21 & Breast cyst fluid & (poorly differentiated) \\
& & Malignant \\
& & (poorly differentiated) \\
\hline
\end{tabular}

Correspondence to: Professor P A Riley

Accepted for publication 29 April 1991 
Table 2 Mean values of malignant specimens $(n=9)$

\begin{tabular}{|c|c|c|c|c|c|c|c|}
\hline $\begin{array}{l}\text { Case } \\
\text { No }\end{array}$ & $\begin{array}{l}\text { Pearson } \\
\text { skewness }\end{array}$ & $\begin{array}{l}\alpha-3 \\
\text { skewness }\end{array}$ & $\begin{array}{l}\text { Line } \\
\text { intercept }\end{array}$ & $\begin{array}{l}\text { Line } \\
\text { slope }\end{array}$ & $C v$ & $\begin{array}{l}\text { Area } \\
\left(\mu^{2}\right)\end{array}$ & $\begin{array}{l}\text { Absorbance } \\
(\lambda=540 \mathrm{~nm})\end{array}$ \\
\hline $\begin{array}{r}1 \\
3 \\
4 \\
6 \\
7 \\
14 \\
18 \\
20 \\
21\end{array}$ & $\begin{array}{l}0.09 \\
0 \cdot 10 \\
0 \cdot 12 \\
0 \cdot 12 \\
0.09 \\
0 \cdot 12 \\
0 \cdot 15 \\
0 \cdot 15 \\
0 \cdot 14\end{array}$ & $\begin{array}{l}1 \cdot 10 \\
1.76 \\
0.52 \\
0.94 \\
1.40 \\
1.03 \\
1.19 \\
0.57 \\
2.03\end{array}$ & $\begin{array}{l}45 \cdot 8 \\
39 \cdot 4 \\
42 \cdot 4 \\
37 \cdot 6 \\
45 \cdot 0 \\
40 \cdot 1 \\
30 \cdot 4 \\
35 \cdot 9 \\
21 \cdot 9\end{array}$ & $\begin{array}{l}28 \cdot 1 \\
13 \cdot 1 \\
43 \cdot 4 \\
47 \cdot 7 \\
24 \cdot 4 \\
42 \cdot 6 \\
65 \cdot 5 \\
51 \cdot 8 \\
33 \cdot 4\end{array}$ & $\begin{array}{l}0 \cdot 62 \\
0 \cdot 36 \\
0 \cdot 70 \\
0 \cdot 62 \\
0.51 \\
0 \cdot 64 \\
0 \cdot 62 \\
0 \cdot 74 \\
0 \cdot 33\end{array}$ & $\begin{array}{l}203 \cdot 4 \\
340 \cdot 7 \\
152 \cdot 0 \\
188 \cdot 7 \\
284 \cdot 5 \\
344 \cdot 6 \\
215 \cdot 7 \\
201 \cdot 1 \\
644 \cdot 8\end{array}$ & $\begin{array}{l}1.61 \\
1.72 \\
1.78 \\
1.43 \\
1.59 \\
2.20 \\
1.36 \\
1.23 \\
1.50\end{array}$ \\
\hline Overall mean (SD) & $0.12(0.02)$ & $1.17(0.50)$ & $37 \cdot 6(7 \cdot 6)$ & $38.9(15.9)$ & $0.57(0.14)$ & $286 \cdot 2(150 \cdot 7)$ & $1.60(0.28)$ \\
\hline
\end{tabular}

(Glen Spectra Ltd, Stanmore, England). Digital video analysis was carried out using a program written in FORTRAN ("CYLUM", copyright DJS) which calculates the pixel absorbances of cells selected by the operator, using procedures described elsewhere (Spargo DJ, Riley PA, unpublished observations). ${ }^{8}$ The set of pixel absorbances for each cell were expressed as a frequency distribution histogram and this distribution was then analysed. Parameters of interest were found to be related to the skewness of the distribution; in particular we examined the Pearson coefficient of skewness, $\alpha-3$ skewness, the slope and intercept of the probit transform of the frequency distribution, and the linear correlation coefficient $(\mathrm{Cv})$ in each case. The area and total absorbance of the cells were also recorded. The individual values for each cell were recorded and the mean values for each sample set calculated.

\section{Results}

Table 1 shows the origin of each specimen and the diagnosis obtained using standard cytological techniques. Nine specimens contained malignant cells; in 12 no malignant cells were seen. For each specimen slides were stained as described and representative fields were selected. All the cells within the selected fields were measured. Several fields were counted until at least 60 cells were included in the sample. A frequency distribution of the pixel absorbances was generated for each cell (fig 1) and statistical parameters were calculated. From the data obtained for individual cells, averages were calculated for each sample, and these are shown for the malignant and non-malignant specimens in tables 2 and 3. Comparisons of these data (table 4) show that with the exception of the intercept of the probit transformed frequency distribution of the absorbance values, the remaining parameters show significant differences between the mean values obtained from the malignant and non-malignant samples. The mean cell size was greater and the mean absorbance higher in the malignant samples. As diagnostic criteria these parameters are likely to be too dependent on the nature of the sample, and absolute absorbance values would be expected to be sensitive to variability in the staining procedure. Because of this we reasoned that the characteristics of the distribution of the point absorbances of a cell might be a more reliable and useful measurement, as this is essentially independent of size and absolute absorbance values. We found that the most powerful discriminants between malignant and nonmalignant samples were the slope of the probit transformation which was substantially greater in non-malignant samples-that is, showing less positive skewness - and the $\alpha-3$ skewness coefficient. Alpha- 3 is the sum of the cubes of the normalised differences between the individual values and the mean of the distribution. Using this test, values above 0.5 indicate positive skewness. ${ }^{9}$ Figure $2 \mathrm{~A}$ shows that the mean values of all the malignant specimens exhibited positive skewness by this criterion; only two of the non-malignant specimens overlap this range-that is, there was a false negative rate of zero and a false positive rate of less than $20 \%$ in our sample. Comparison of the slopes for the linearised frequency distribution also gives quite good discrimination, but the overlap was slightly larger between the malignant and non-malignant samples (fig 2B). The significantly lower correlation coefficient of

Table 3 Mean values of non-malignant specimens $(n=12)$

\begin{tabular}{|c|c|c|c|c|c|c|c|}
\hline $\begin{array}{l}\text { Case } \\
\text { No }\end{array}$ & $\begin{array}{l}\text { Pearson } \\
\text { skewness }\end{array}$ & $\begin{array}{l}\alpha-3 \\
\text { skewness }\end{array}$ & $\begin{array}{l}\text { Line } \\
\text { intercept }\end{array}$ & $\begin{array}{l}\text { Line } \\
\text { slope }\end{array}$ & $C v$ & $\begin{array}{l}\text { Area } \\
\left(\mu^{2}\right)\end{array}$ & $\begin{array}{l}\text { Absorbance } \\
(\lambda=540 \mathrm{~nm})\end{array}$ \\
\hline $\begin{array}{r}2 \\
5 \\
8 \\
9 \\
10 \\
11 \\
12 \\
13 \\
15 \\
16 \\
17 \\
19\end{array}$ & $\begin{array}{l}0 \cdot 10 \\
0 \cdot 13 \\
0 \cdot 15 \\
0 \cdot 16 \\
0 \cdot 12 \\
0 \cdot 14 \\
0 \cdot 14 \\
0 \cdot 10 \\
0 \cdot 15 \\
0 \cdot 28 \\
0 \cdot 28 \\
0 \cdot 29\end{array}$ & $\begin{array}{r}0.58 \\
-0.03 \\
-0.08 \\
-0.06 \\
0.34 \\
0.48 \\
0.46 \\
0.36 \\
0.63 \\
0.22 \\
0.05 \\
0.34\end{array}$ & $\begin{array}{l}45 \cdot 6 \\
42 \cdot 3 \\
39 \cdot 7 \\
38 \cdot 5 \\
45 \cdot 6 \\
38 \cdot 6 \\
38 \cdot 8 \\
56 \cdot 3 \\
34 \cdot 1 \\
24 \cdot 7 \\
21 \cdot 2 \\
21.5\end{array}$ & $\begin{array}{r}48.9 \\
83.0 \\
81.0 \\
86.4 \\
71.3 \\
48.7 \\
65.7 \\
55.0 \\
68.9 \\
82.4 \\
109.3 \\
105.8\end{array}$ & $\begin{array}{l}0 \cdot 76 \\
0 \cdot 88 \\
0 \cdot 88 \\
0 \cdot 89 \\
0 \cdot 82 \\
0 \cdot 81 \\
0.80 \\
0.76 \\
0 \cdot 77 \\
0 \cdot 85 \\
0.88 \\
0.86\end{array}$ & $\begin{array}{r}129 \cdot 5 \\
86.7 \\
72 \cdot 5 \\
79 \cdot 4 \\
59 \cdot 6 \\
50 \cdot 2 \\
66 \cdot 7 \\
57 \cdot 1 \\
78 \cdot 8 \\
100 \cdot 1 \\
46 \cdot 5 \\
86.5\end{array}$ & $\begin{array}{l}1.47 \\
1.34 \\
1.40 \\
1.07 \\
1.47 \\
1.61 \\
0.97 \\
1.19 \\
1.30 \\
1.07 \\
1.15 \\
1.27\end{array}$ \\
\hline Overall mean $(S D)$ & $0.17(0.07)$ & $0.27(0.25)$ & $37 \cdot 2(10 \cdot 5)$ & $75 \cdot 5(19 \cdot 8)$ & $0.83(0.05)$ & $76 \cdot 1(23 \cdot 3)$ & $1 \cdot 28(0 \cdot 19)$ \\
\hline
\end{tabular}


Table 4 Comparison of parameters (means SD)

\begin{tabular}{lccll}
\hline & Malignant $(n=9)$ & Non-malignant $(n=12)$ & $t$ & $p$ \\
\hline Pearson skewness & $0.12(0.02)$ & $0.17(0.07)$ & 2.50 & $<0.05$ \\
o-3 skewness & $1.17(0.50)$ & $0.27(0.25)$ & 5.29 & $<0.001$ \\
Line intercept & $37.6(7.6)$ & $37 \cdot 2(10.5)$ & 0.10 & $>0.2$ \\
Line slope & $38.9(15.9)$ & $75.5(19.8)$ & 4.55 & $<0.001$ \\
Cv & $0.57(0.14)$ & $0.83(0.05)$ & 2.60 & $<0.02$ \\
Area & $286.2(150.7)$ & $76.1(23.3)$ & 4.80 & $<0.01$ \\
Absorbance & $1.60(0.28)$ & $1.28(0.19)$ & 3.20 & $<0.01$ \\
\hline
\end{tabular}

the linearised frequency distribution for the malignant samples (table 4 ) is not unexpected, because the linearising transformation depends on the assumption of a "normal"-non-skewed distribution. ${ }^{10}$

\section{Diścussion}

To design a system which reads cytological material in the same way as human experts is very difficult, due to the problem of simulating the complex spatial and textural clues involved in the interpretation of visual input. In this study we used an alternative analytical approach by identifying featureless criteria which have the power to discriminate between malignant and non-malignant phenotypes. These parameters are not consciously perceived by human observers, but are much easier to generate and manipulate using automated techniques than the visual cues used by people.

The preliminary data reported here indicate that it may be practical to use simple numerical criteria as diagnostic indicators in cytology, by treating cells as a set of localised point absorbances. The use of the characteristics of a non-cognitive absorbance pattern seems intrinsically to have several important advantages over the analysis of morphological features or absolute absorbances, because it does not depend on the orientation or the detailed morphology of the cells and is also essentially independent of magnification and other conditions that could modify the absolute absor-
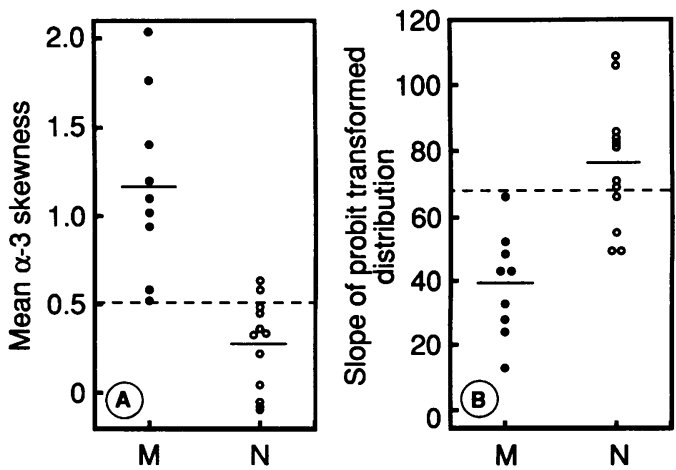

Figure 2 Mean values of $(A) \alpha-3$ skewness of the frequency distribution of pixel absorbances and $(B)$ the slope of the linearised frequency distribution obtained in the case of malignant $(M)$ and non-malignant $(N)$ material. The horizontal bars indicate the overall means in each class. The interrupted line signifies: $(A)$ the minimum value for positive skewness $(\alpha-3>0.5)$;

$(B)$ an arbitrary upper threshold of the slope of the linearised frequency distribution which would include all malignant cases.

bance values. The system is also largely independent of optical resolution provided that this is greater than that of any subset of absorbing features in the object, although the accuracy of estimates based on the frequency distribution will clearly increase with increasing spatial resolution as the sample size is increased. Our data show that malignant cells show a significant positive skewness of the distribution of point absorbances which reflects differences in their staining characteristics. Because the method analyses the skewness parameters of the pixel absorbances of each object in the delineated field, the procedure could be used on groups or clumps of cells. This has the disadvantage, however, of averaging the pixel absorbances of several cells which may, if the clump contains several normal cells, obscure the skewness pattern of a single abnormal cell, which in principle would otherwise be detectable by this method.
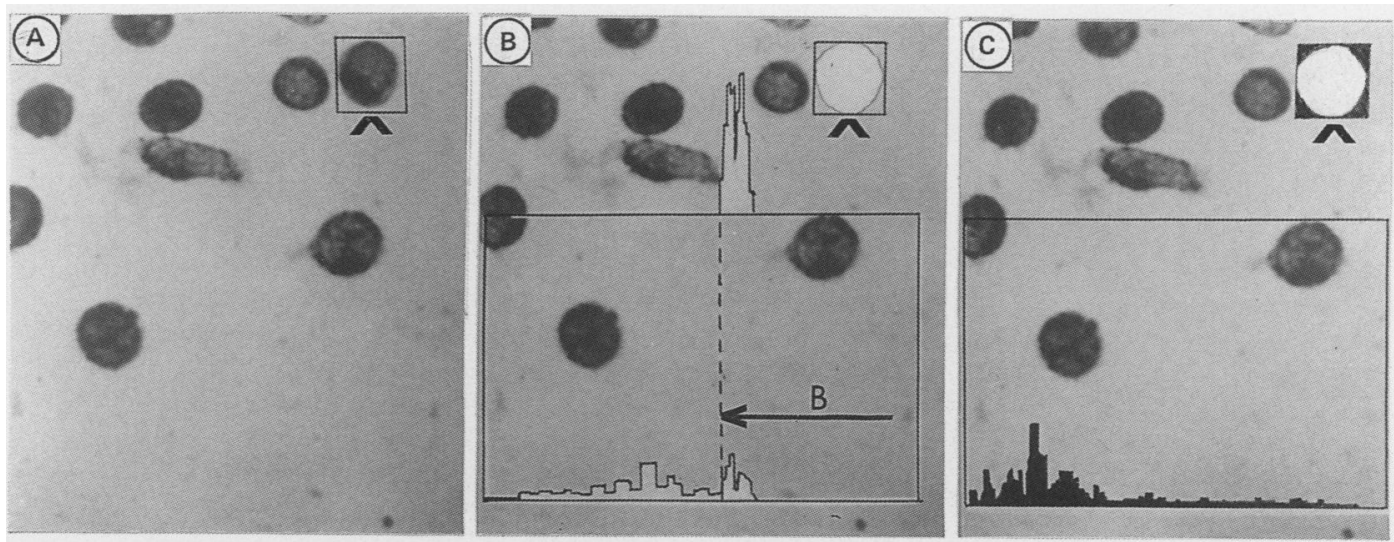

Figure 1 Illustration of the procedure for processing the data from a digitised image of a cell.

(A) The cell to be measured is identified by a box as indicated in the upper right quadrant of the field illustrated. (B) The frequency distribution of the luminosities of the pixels in the area delineated by the box is displayed in the lower panel (ordinate: frequency; abcissa: luminosity) and compared with the frequency distribution of the luminosities of the equivalent background field (shown in the upper panel) from which the threshold value (interrupted line) is derived. Pixels with luminosities greater than this threshold value (arrowed) are excluded from the analysis as background pixels $(B)$.

(C) The individual pixel absorbances of the defined object are calculated by comparing the pixel luminosities of the object with the luminosities of pixels with identical locations in a blank reference image stored in the frame store of the computer. The frequency distribution of these point absorbances of the cell are displayed as a histogram in the lower panel (ordinate: frequency; abcissa: absorbance). Statistical analysis of this frequency distribution was made for each cell in a sample as described in the text. 
In view of the observed false negative rate of zero the technique in its present form might provide the basis of an automated pre-screening method. The system requires refinement to reduce the unacceptably high false positive rate of $20 \%$, however, and wider application would depend on its performance in a larger series of tests encompassing material from different sources and different staining methods.

We conclude that skewness parameters may be of value as a diagnostic criterion in the detection of malignancy in cytological specimens. Because of its relative simplicity, this method of discrimination may prove useful for use in automated cytological screening procedures.

We are indebted to Dr Chandra Grubb for supplying the cytological specimens. The work was supported by the Association for International Cancer Research.
1 Baak JPA, Van Diest PJ, et al. The multicenter morphometric mammary carcinoma project. A nationwide prospective study on reproducibility and prognostic power of quantitative assessments in The Netherlands. Path Res Pract 1989;185:664-70.

2 Bartels PH. Numerical evaluation of cytological data, VI. Multivariate distributions and matrix notations. Analyt Quant Cytol 1980;2:155-60.

3 Tranzer JP, Pearse AGE. Cytochemical demonstration of ubiquinones in animal tissues. Nature 1963;199:1063-6.

4 Pearse AGE. Coenzymes. In: Histochemistry, theoretical and applied. 3rd ed. Edinburgh: Churchill Livingstone, 1972: $1000-4$.

5 Lee JA. Histochemical detection of ubiquinone in neutrophil polymorphonuclear leucocyte granules. Exp Cell Biol 1986;54:89-93.

6 Pedersen PL. Tumour mitochondria and the bioenergetics of cancer cells. Progr Exp Tumour Res 1978;22:190-274.

7 Wilkie D, Evans IH, Egilsson V, Diala ES, Collier D. Mitochondria, cell surface and carcinogenesis. Int Rev Cytol 1984;15(Suppl):157-89.

8 Spargo DJ, Riley PA. Computerized digital video microdensitometry applied to the quantitation of intracellula macromolecules or their functional groups. In: McBrien $\mathrm{DCH}$, Slater TF, eds. Eicosanoids, lipid peroxidation and cancer. Berlin: Springer-Verlag, 1988:315-25.

9 Parsons R. Statistical analysis: a decision-making approach. New York: Harper and Row, 1974.

10 Colquhoun D. Lectures on Biostatistics. Oxford: Clarendon Press, 1963. 